\title{
Energy-aware Virtual Ring Routing in Wireless Sensor
}

\section{Networks}

\author{
Ghofrane FERSI, Wassef LOUATI, Maher BEN JEMAA \\ Research Unit of Development and Control of Distributed Applications (ReDCAD) \\ Department of Computer Science and Applied Mathematics \\ National School of Engineers of Sfax, BP 1173-3038 Sfax, Tunisia \\ University of Sfax
}

ghofrane.fersi@acm.org, wassef.louati@redcad.org,maher.benjemaa@enis.rnu.tn

Received: December 1, 2010 Accepted: December 31, 2010 DOI: 10.5296/npa.v2i4.494

\begin{abstract}
Virtual Ring Routing (VRR) is an ad-hoc routing protocol that avoids flooding the network. It uses location independent identifiers for packet routing. However, VRR does not take into consideration the amount of energy in nodes, leading to the failure of several nodes in the network. To resolve this problem, we have adopted a hierarchical Distributed Hash Table in the VRR architecture. In the resulting architecture, the energy powerful sensors are responsible for most message traffic. Weak sensors are then utilized less frequently than powerful sensors. The experimental results show that Coral-based VRR consumes less energy than VRR and extends the network lifetime.
\end{abstract}

Keywords: Wireless Sensor Networks, Energy-aware routing, Virtual Ring Routing, hierarchical Distributed Hash Table 


\section{Introduction}

Recent advances in Wireless Sensor Networks (WSNs) have motivated the development of specific routing protocols. Energy awareness is very crucial in the development of these protocols [1] [2]. In fact, the amount of energy in sensor nodes is so critical that energy costeffectiveness becomes the most important constraint in WSNs.

Several routing protocols have been developed to solve WSNs challenges. They are classified into different classes [3]. Data centric protocols [4] use advertisements before data transmission in order to avoid redundancy. Hierarchical protocols [5] classify nodes into clusters and perform large hops in each cluster. Cluster heads perform some message aggregation in order to save energy. Location-based protocols [6] use the position information in order to forward data to the final destination. Identity-based protocols ensure routing on the identities themselves without having location information in the packet header.

VRR [7] is the first scalable identity-based protocol. It has a design inspired by Distributed Hash Table (DHT) overlays. VRR avoids flooding the network and organizes nodes into a virtual ring ordered by their location independent identifiers. Routing is achieved using only these semantic-free identifiers. However, VRR does not take into consideration the amount of energy in nodes, leading to frequent node failure.

To resolve this problem, this paper proposes a hierarchical VRR based on nodes energy amounts. The hierarchy structure is inspired by Coral [8], a hierarchical DHT system. The proposed architecture, called Coral-based VRR, classifies the nodes in a hierarchical manner, for example, in three nested virtual rings (the regular peers ring, the superpeers ring and the hyperpeers ring), according to their amount of energy. Routing in each ring is performed by the VRR protocol. To send a packet from a node to another, the packet uses mainly the hyperpeers ring. The others rings are just used to reach the hyperpeers ring. In this manner, powerful nodes are more solicited than others nodes, leading to a longer network lifetime. The proposed approach is evaluated by simulations and compared to VRR. The experiments show that our approach outperforms VRR in terms of energy consumption, end-to-end delay, routing performance and network lifetime.

The remainder of this paper is organized as follows. Section 2 gives an overview of the VRR protocol. Section 3 describes the proposed Coral-based VRR approach. Simulation results are reported in section 4 . Section 5 presents the related work and finally section 6 concludes the paper.

\section{Virtual Ring Routing}

VRR is a scalable identity-based routing protocol. Nodes have location-independent identifiers and are organized into a virtual ring ordered by their identifiers. Fig. 1 shows the mapping between physical and logical topology in a virtual ring.

Each node in VRR maintains in its routing table:

- A virtual neighbor set (called also endpoints) containing generally the node identifiers of 2 closest neighbors clockwise in the virtual ring and 2 closest neighbors counter clockwise. Paths to these nodes are called vset-paths.

- A physical neighbor set containing the identifiers of the physical neighbors towards virtual neighbors. 
To forward messages from a node to another, VRR chooses the node having the closest identifier to the destination, and forwards the message to its corresponding next physical hop. The nodes along a routing path store the next hop towards each path endpoint in a routing table.

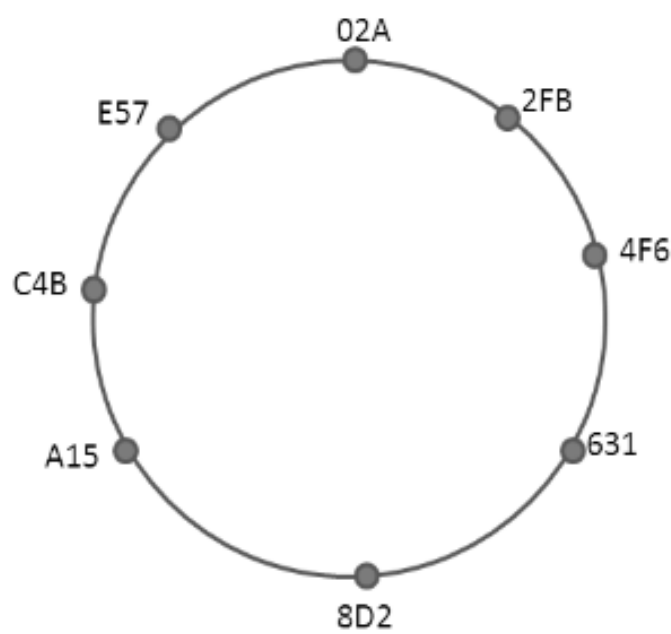

Virtual topology

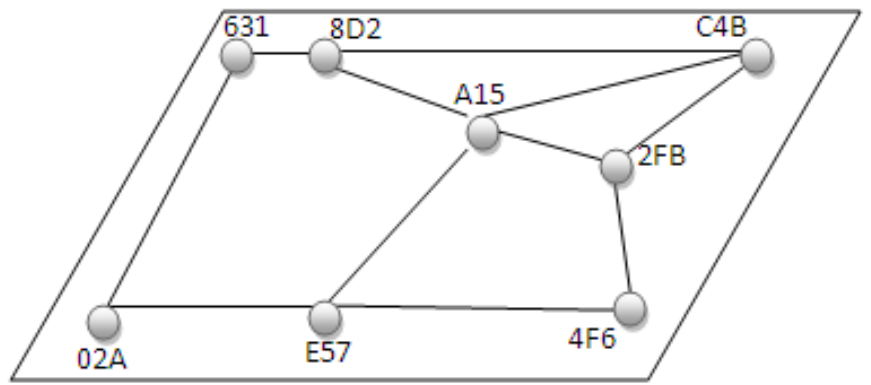

Physical topology

Figure 1. Virtual and physical structure in VRR

Table 1 shows the routing table of the node C4B. The first two entries are vset-paths to C4B's virtual neighbors (E57 and 02A). The third entry is an example of a virtual path in which the node $\mathrm{C} 4 \mathrm{~B}$ participated in its construction without being one of its endpoints. The three last entries are paths to the C4B's physical neighbors.

Table1.Sample routing table for the node C4B

\begin{tabular}{|c|c|c|c|c|}
\hline Endpoint A & Endpoint B & Next A & Next B & Path id \\
\hline E57 & C4B & 2FB & NULL & 04 \\
\hline $02 \mathrm{~A}$ & C4B & $8 \mathrm{D} 2$ & NULL & 10 \\
\hline $4 \mathrm{~F} 6$ & 631 & $2 \mathrm{FB}$ & $8 \mathrm{D} 2$ & 07 \\
\hline C4B & $8 \mathrm{D} 2$ & NULL & $8 \mathrm{D} 2$ & FF \\
\hline C4B & A15 & NULL & A15 & FF \\
\hline C4B & 2FB & NULL & 2FB & FF \\
\hline
\end{tabular}


VRR is scalable and supports mobility and fault tolerance. However, VRR is not an energy-aware routing protocol. In the next section, we propose Coral-based VRR that classifies the nodes in a hierarchical manner depending on their amount of energy. Packets are routed by doing large hops towards the nodes having an important amount of energy, while avoiding poor energy nodes.

\section{Coral-based Virtual Ring Routing}

\subsection{Coral}

Coral is a hierarchical DHT system that classifies the nodes in different levels. Nodes in each level are organized into a virtual ring. Each level has a subset of the nodes belonging to the previous level: the first level, also called the highest level, includes all nodes; the second level is a subset of the first level (previous level) and so on. This hierarchical classification enables a rapid data lookup. Indeed, packets are routed mostly in the lower level ring (that is holding the minimal number of nodes). This enables large routing hops and consequently minimizes the data lookup cost. For example, in fig.2, to find a key (it can be a file URL), node $\mathrm{r}$ starts the search in the lowest level (arrows number 1 and 2), and since the node $\mathrm{t} 2$ does not store the key and this node is the closest one to the key in the lowest level, the lookup continues on the higher level (arrows number 3 and 4) until finding the node having the key (the node t0).

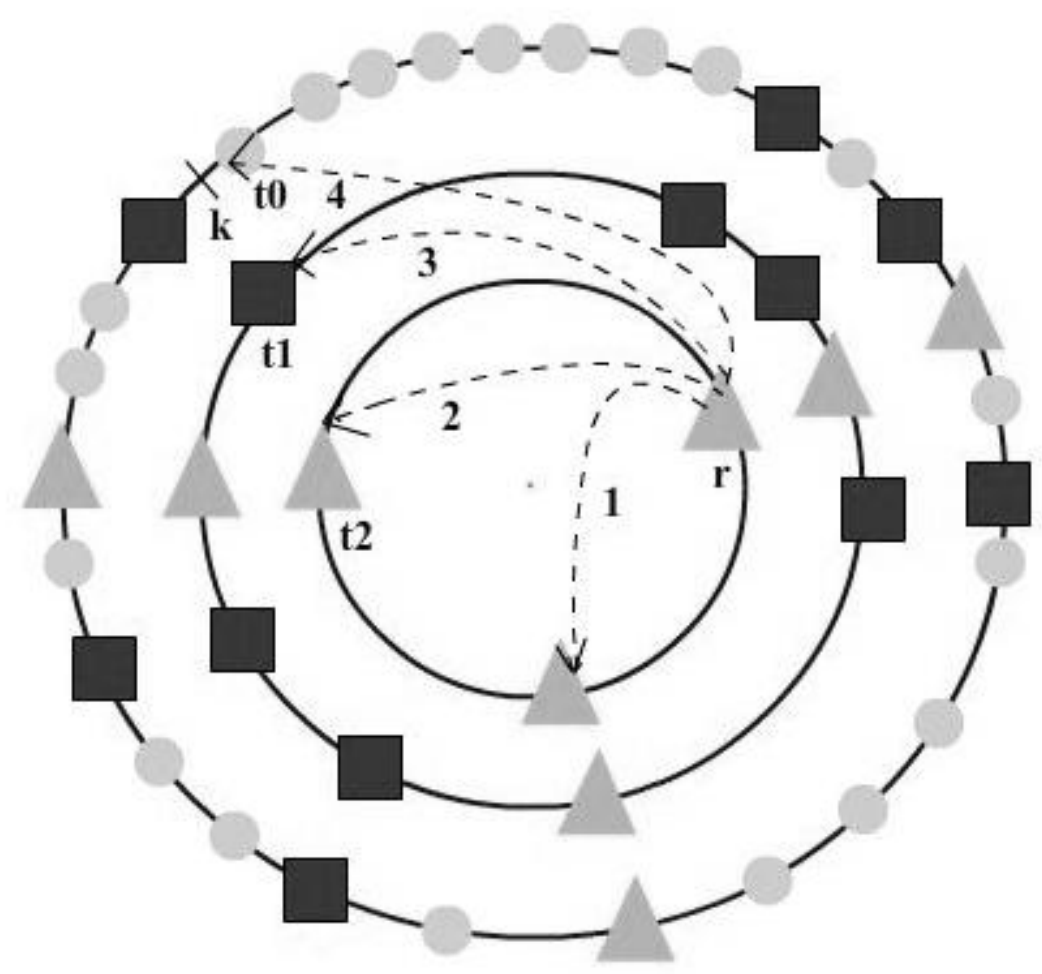

Figure 2. Coral architecture

\subsection{Overview of the proposed Coral-based VRR architecture}

In Coral-based VRR, nodes are classified into three classes: 
- Peers are the nodes having a critical amount of energy (for example less than 3 Joules).

- Superpeers are the nodes having more energy than peers (for example between 3 and 5 Joules).

- Hyperpeers are the nodes having a big amount of energy (for example more than 5 Joules).

These nodes are structured hierarchically like in Coral. The first level in Coral-based VRR includes all types of nodes: peers, superpeers and hyperpeers. The second level excludes the peers having critical amount of energy (according to a given threshold), thus containing only superpeers and hyperpeers nodes. The third level includes only hyperpeers, the most powerful nodes in the network.

\subsection{Node joining}

When a node joins the network, it sets its type (a peer, a superpeer, or an hyperpeer) according to a superpeer-specific threshold and a hyperpeer-specific threshold. If the joining node is a regular peer, it should be positioned only in the first ring (peers ring). But if the node is a superpeer, it should be positioned in the peers ring and the superpeers ring. Otherwise, it should search its place in the three rings. To perform the joining procedure, Coral-based VRR uses different types of messages:

- Hello messages are used to find physical neighbors.

- Setup-request messages search the virtual neighbours peers, virtual neighbors superpeers and virtual neighbors hyperpeers of the joining node.

- Setup messages are routed back to the joining node in order to inform it of its virtual neighbours peers, superpeers and hyperpeers. These messages are used to setup the virtual paths between the source and the destination.

For example, a network of 8 nodes which have the following identifiers: 02A, 2FB, 4F6, $631,8 \mathrm{D} 2, \mathrm{~A} 15, \mathrm{C} 4 \mathrm{~B}, \mathrm{E} 57$. The physical structure of this network is given in fig.1. Nodes having the identifiers $02 \mathrm{~A}, \mathrm{~A} 15,4 \mathrm{~F} 6$ are superpeers, the other nodes are peers. When the superpeer 632 joins the network, the following steps occur:

1) The joining node applies the VRR joining process in order to be well positioned in the peers ring (between the nodes having the identifiers 631 and 8D2).

2) The virtual neighbor 631 forwards the setup-request message to its virtual neighbour $8 \mathrm{D} 2$.

3) The node having the identifier $8 \mathrm{D} 2$, forwards the setup-request message to the next virtual hop A15.

4) A15 receives the setup-request message, and since A15 is a superpeer, it does not forward the message to the next virtual neighbor peer C4B, but it relays the message to the superpeers ring and applies the VRR joining procedure in the superpeers ring. At the end of these steps, the node with identifier 632 is well positioned in the superpeers ring.

Algorithm 1 presents a pseudo code of the node joining process in the proposed approach. 


\section{Algorithm 1 Coral-based VRR node joins pseudo code}

1: if energy $>=$ threshold 1 then

2: status= hyperpeer

3: else

4: $\quad$ if energy $>=$ threshold 2 then

5: $\quad$ status $=$ superpeer

6: else

7: $\quad$ status $=$ peer

8: VRR-Process (RegularPeersLevel, NodeId)

9: Receive setup-request (message)

10: if status $=$ peer then

11: $\quad$ VRR-Process (RegularPeersLevel, NodeId)

12: else

13: $\quad$ if status $=$ superpeer then

14: $\quad$ if IamNearestHop(message.nodeId) $=$ false then

15: $\quad$ VRR-process (SuperpeersLevel, NodeId)

16: else

17: $\quad$ if message.level=peer then

18: $\quad$ VRR-process (RegularPeersLevel, NodeId)

19: $\quad$ else

20: $\quad$ if message.level=superpeer then

21: $\quad$ Send-setup (me, nodeId, nh, nVirt, 2, Superpeer, VsetSupepeer )

22: $\quad$ else

23: $\quad$ Send-setup (me, nodeId, nh, nVirt, 3, Superpeer, VsetSupepeer)

24: $\quad$ Send-setup-request (me, nodeId, nh, me.Succ, 3, hyperpeer)

25: else

26: $\quad$ if IamNearestHop(message.nodeId $)=$ false then

27: $\quad$ VRR-process (HyperpeersLevel, NodeId)

28: $\quad$ else

29: $\quad$ if message.level=hyperpeer then

30: $\quad$ Send-setup (me, nodeId, nh, nVirt, 3, Hyperpeer, VsetHyperpeer)

31: $\quad$ else

32: $\quad$ VRR-process (Superpeerslevel, NodeId)

In this algorithm, $\boldsymbol{m} \boldsymbol{e}$ is the identifier of the local node, $\boldsymbol{n h}$ is the next physical hop, $\boldsymbol{n V i r t}$ is the next virtual hop, NodeId is the identifier of the joining node, Succ is the immediate successor node, VRR-process(RegularPeersLevel, NodeId), VRR-process(SuperpeersLevel, NodeId) and VRR-process(HyperpeersLevel, NodeId) apply the VRR signaling procedure respectively in the first ring (peers ring), in the second ring (superpeers ring) and in the third and final ring (hyperpeers ring), the message level gives the current step in the joining procedure. IamNearestHop () is a boolean function that returns true if the current node is the nearest one to the destination. VsetSuperpeer and VsetHyperpeer are respectively the set of virtual neighbours superpeer and the set of virtual neighbors hyperpeer of the source node that sent the setup message.

\subsection{Data packet routing}

Coral-based VRR routes data packets to destination identifiers as follows. When a packet is received by a regular peer, the packet is forwarded using the VRR forwarding algorithm, 
until to find the destination identifier or to find a superpeer. In the second case, the packet is routed in the superpeers ring using the VRR forwarding algorithm, until to find the destination identifier, or to find a hyperpeer. If it finds an hyperpeer, the VRR forwarding algorithm is used in the hyperpeers ring until reaching the destination identifier in that ring. If there is not such hyperpeer, the packet descends to the superpeers level, then to the regular peers level, until reaching the destination identifier. For example, the fig. 3 shows how a packet is transmitted from the node 2 to the node 40, using the proposed routing approach. The figure shows also the path of the same packet using the VRR approach. Compared to VRR, the proposed approach performs clearly less hops toward the destination node (10 hops with VRR and only 6 hops with Coral-based VRR).
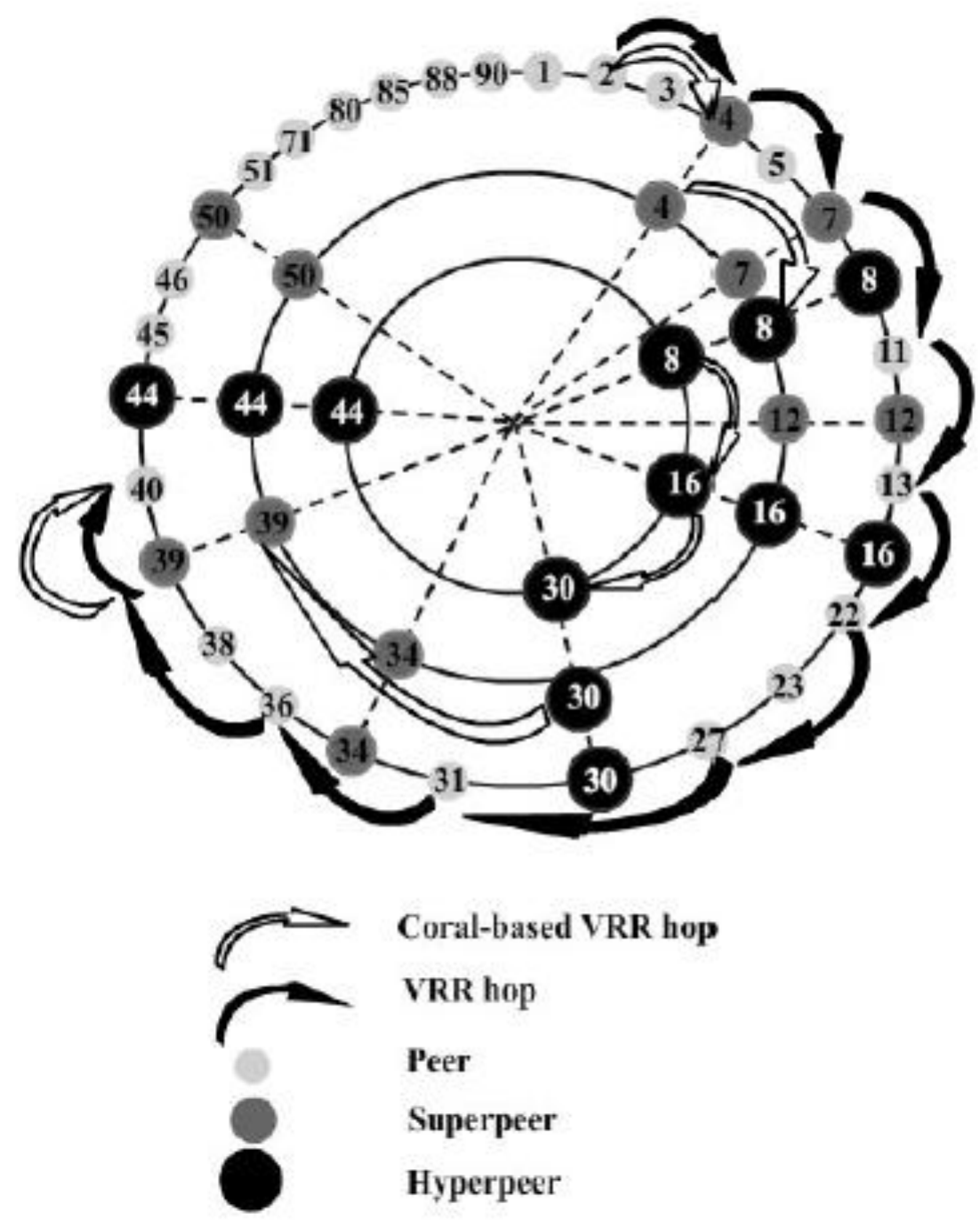

Figure 3. Coral-based VRR architecture

Algorithm 2 explains the data packet routing in Coral-based VRR.

\section{Algorithm 2 Pseudo code for Coral-based VRR data routing}

1: Receive datapacket (packet)

2: if status=peer then 
3: VRR-Routing-process (RegularPeersLevel, destination)

4: else

5: $\quad$ if status $=$ superpeer then

6: $\quad$ if IamNearestHop (destination) $=$ false then

7: $\quad$ VRR-Routing-process (SuperpeersLevel, destination)

8: $\quad$ else

9: $\quad$ VRR-Routing-process (RegularPeersLevel, destination)

10: else

11: $\quad$ if IamNearestHop (destination) $=$ false then

12: VRR-Routing-process (HyperpeersLevel, destination)

13: else

14: $\quad$ VRR-Routing-process (SuperpeersLevel, destination)

VRR-Routing-process (RegularPeersLevel, destination), VRR-Routing-process (SuperpeersLevel, destination), VRR-Routing-process (HyperpeersLevel, destination) apply the VRR routing process respectively in the regular peers ring, in the superpeers ring and in the hyperpeers ring. IamNearestHop (destination) is a boolean function which indicates that the current node is the closest node to the destination in the actual ring or not.

\subsection{Node and link failures}

Nodes mobility or failures make a lot of changes in the network topology, but these changes shouldn't affect the network consistency. All reparations and updates should be done in a timely fashion. In Coral-based VRR, the failure detection and repair can be performed in a similar way as in VRR. The routing of signaling messages, such as teardown messages which are sent when a node is no longer a virtual neighbour of another node, is performed by the proposed Coral-based VRR routing process.

\subsection{Node dynamic state}

Since hyperpeers are more solicited than other nodes in Coral-based VRR, their amounts of energy will be degraded rapidly and reach the superpeer threshold. In this case, an hyperpeer leaves the hyperpeers ring and will belong only to the superpeers ring and the peers ring. Similarly, if the amount of energy of a given superpeer reaches the peers threshold, the said superpeer leaves the superpeers ring and will belong only to the peers ring. Hence, after a while, all hyperpeers and superpeers will disappear and all nodes become regular peers. The advantage here is that we have exploited the energies of the most powerful nodes before their leaving.

\section{Simulation}

We have evaluated the proposed Coral-based VRR using the NS-2.33 simulator [9], supporting sensor networks simulations. The simulation compares the performance of Coralbased VRR system to the VRR system. Nodes are placed randomly and organized into two levels: the peers ring and superpeers ring. The superpeers have an initial amount of energy equal to 5 Joules and the regular peers have an initial amount of energy equal to 3 Joules. All nodes have the same transmission power $\left(8.5872 * 10^{-4} \mathrm{~W}\right)$, and their transmission range is 40 meters.

A first experiment evaluates the routing performance. It determines the number of contacted nodes (for packets routing) according to the network size. Fig.4 shows that Coral- 
based VRR outperforms VRR for all network sizes. In Coral-based VRR, packets perform large virtual hops toward their destinations thanks to the hierarchical structure of Coral-based VRR.

A second experiment determines the average latency time of the packets routing according to the network size. Fig. 5 shows that Coral-based VRR achieves low delays since it performs few logical hops toward packets destinations.

A third experiment measures the energy consumption variation along the time in a network of 30 nodes. A FTP traffic is generated in the network. Fig. 6 shows that Coral-based VRR consumes globally less energy than VRR. In the setup phase (until 900 seconds), the Coral-based VRR consumes more energy than VRR due to the routing of extra messages for the superpeer joining process. In the packets routing phase, Coral-based VRR consumes clearly less energy than VRR since VRR contacts more nodes. Furthermore, this energy optimization increases the network lifetime. Fig.7 shows that the number of dead nodes in VRR increases more rapidly than in Coral-based VRR, since our approach avoids sending messages to critical energy nodes.

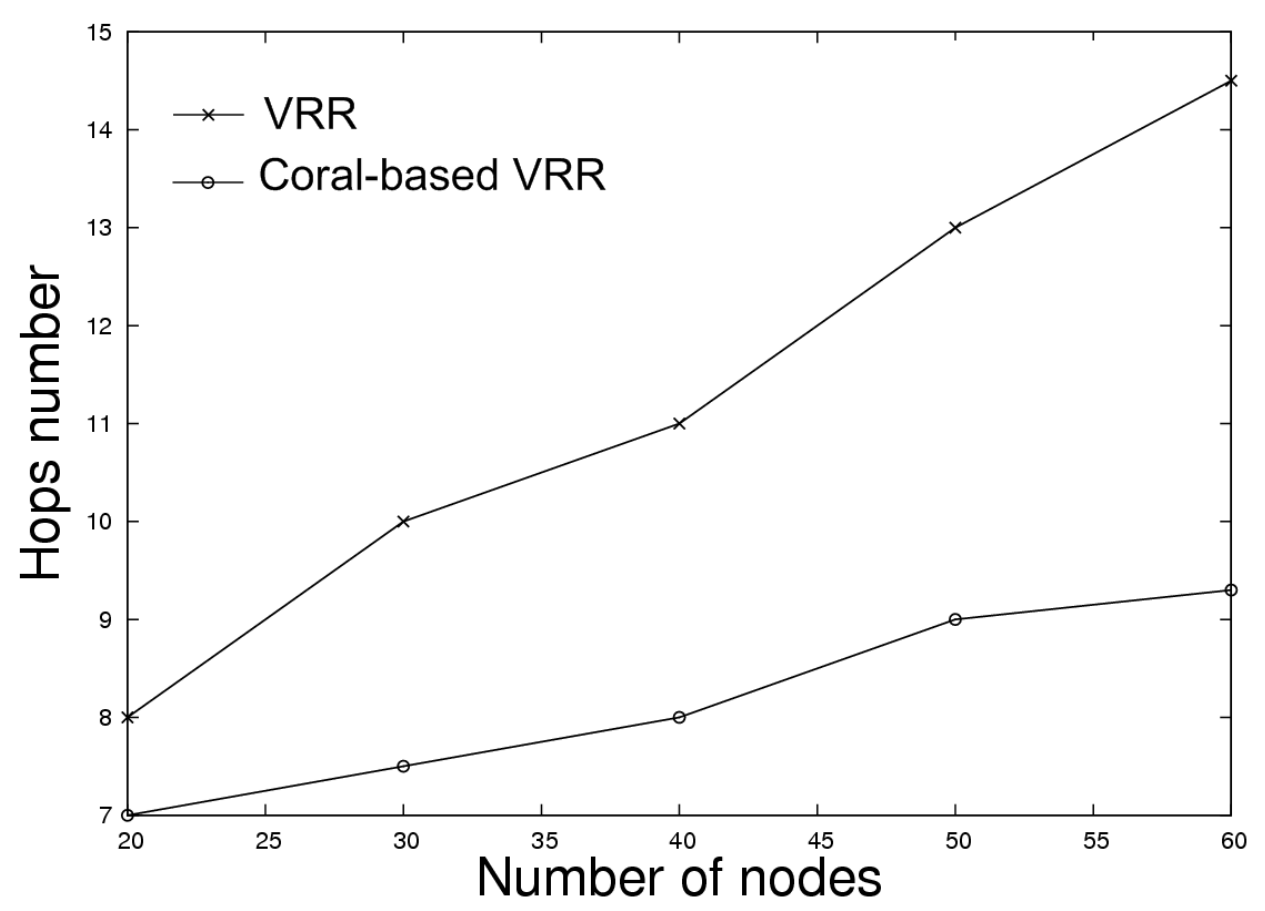

Figure 4. Routing performance 


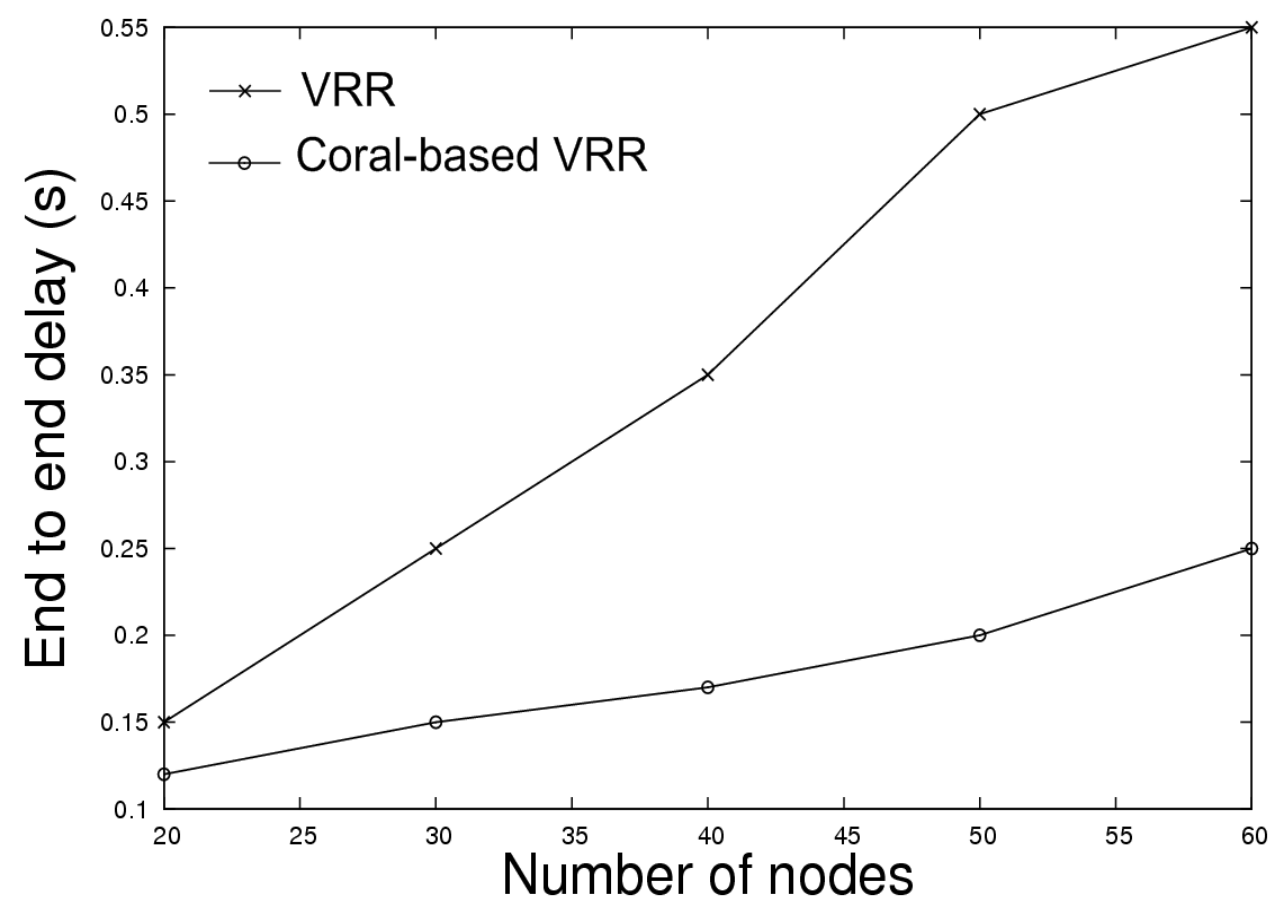

Figure 5. Average end-to-end delay

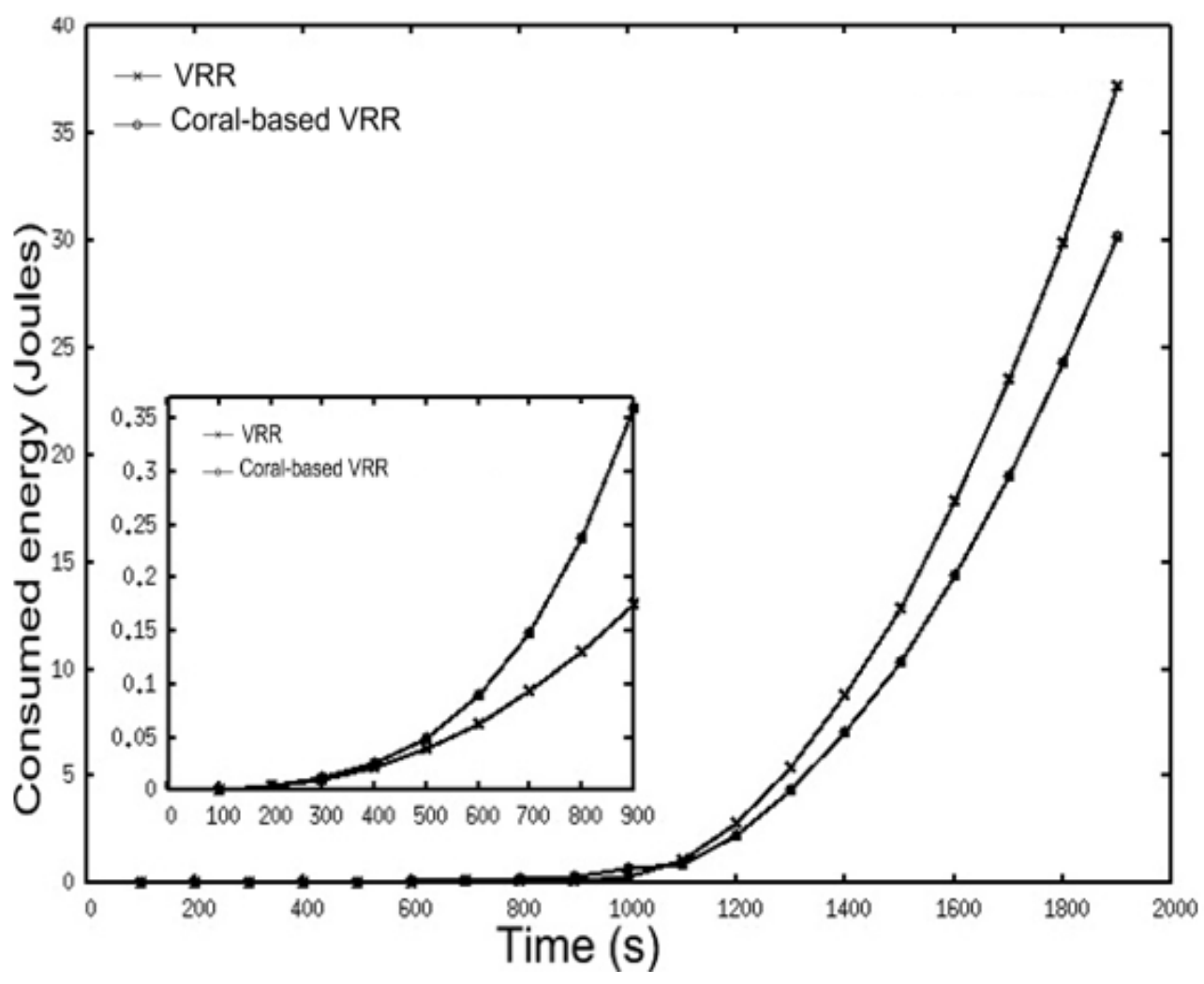

Figure 6. Variation of energy consumption along the time 


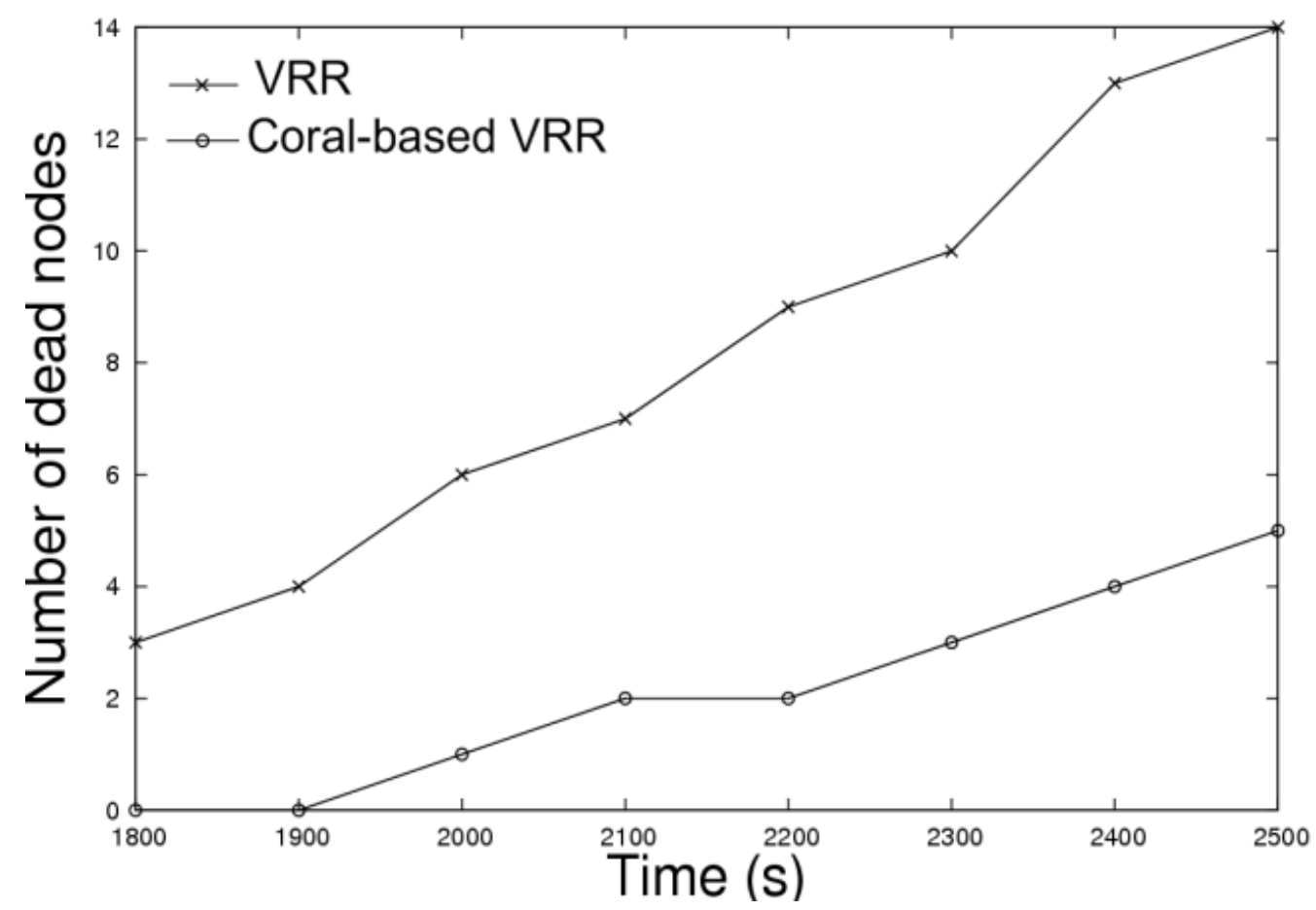

Figure 7. Network lifetime

\section{Related work}

\subsection{DHT based routing protocols in wireless sensor networks}

We can classify DHT based approaches in Wireless Sensor Networks in 3 classes: reallocation based, virtual location based and location independent [10]. Geographic Hash Tables (GHT) [11] is a real-location based protocol that looks at a sensor network as a distributed database. GHT hashes keys into geographic locations so that a data item is stored on the sensor node geographically nearest to the hash of its corresponding key. GHT is simple and provides good load balancing in storage. Coral-based VRR manages successfully nodes mobility while GHT presents some weaknesses when nodes move or disconnect.

Geographic Routing Without Location Information (GRWLI) [12] is a virtual coordinate based protocol. Its architecture consists of a n-dimensional virtual coordinate system. The virtual coordinates for nodes are based essentially on the distance and the number of hops between the nodes. The main limit of this routing protocol is that the virtual coordinate assignment requires very long time to converge which lead to a further energy consumption, while in Coral-based VRR, the virtual identifiers assignment do not require any additional work, and can simply be the MAC addresses, which would preserve the energy consumption.

Tiered Chord (TChord) [13] is a simplified mapping of Chord on sensor networks. Sensor nodes are organized into a ring and all messages are routed clockwise. In every ring, there is at least one master node with a high power. This master node has information about all its slave nodes and $\mathrm{O}(\log \mathrm{N})$ other master nodes in its local finger table. So, the master node will be more solicited in the routing than slave nodes. The main advantage of this approach is that it takes into account the diversity of nodes characteristics in the routing. However, the routing 
table size of master nodes can increase significantly with increasing network size. In Coralbased VRR, routing tables do not depend on network size.

Virtual Cord Protocol (VCP) [14] is a virtual location based and DHT-like protocol. VCP combines successfully routing techniques as well as DHT services. However, VCP does not take into account the node characteristics.

\subsection{Energy-aware routing protocol}

There are a tremendous number of proposed energy-aware routing protocols for wireless sensor networks. We focus on the most popular ones.

Low Energy Adaptive Clustering Hierarchy (LEACH) [5] forms clusters and uses the cluster heads as routers to the sink. All data compressions are done in every cluster. LEACH minimizes significantly energy consumption and is completely distributed. However, it uses one hop routing from the cluster head to the sink. This makes it inapplicable in extended regions.

Power-efficient Gathering in Sensor Information Systems (PEGASIS) [15] is a LEACH enhancement. In PEGASIS, nodes communicate only with their closest nodes by forming chains at the place of clusters. Only one sensor from the chain transmits to the base station. PEGASIS optimizes more effectively power consumption than LEACH since it avoids the LEACH overhead caused by dynamic cluster formation. PEGASIS reduces the number of transmissions and receptions thanks to data aggregation.

Hierarchical-PEGASIS [16] is an extension to PEGASIS that aims to reduce the transmission delay and to gather data effectively by considering the metric energy $*$ delay.

Threshold sensitive Energy Efficient sensor Network protocol (TEEN) [17] is designed for the sudden changes in the sensed attributes by the use of two limits: soft and hard thresholds: The hard threshold is the minimal value of the attribute that the sensor should transmit to the cluster head. If the sensor senses only the values beyond the hard threshold, it doesn't transmit data only if the difference between the two sensed attributes is equal or greater than the soft threshold. This approach reduces significantly the number of transmitted messages.

Adaptative Threshold sensitive Energy Efficient sensor Network protocol (APTEEN) [18] is a TEEN extension. When the base station forms the clusters, the cluster heads broadcast three types of information: the attributes, the thresholds values, transmission time slots.

In Self organizing protocol [19], sensor nodes are heterogeneous and can be mobile or static. Some sensor nodes collect data and send it to router nodes. Gathered data are sent to the sink nodes. The use of self organizing protocol offers a very low cost of routing table maintenance and also it is fault tolerant.

Energy Aware Intra Cluster Routing (EAICR) [20] aims at optimizing energy consumption for the closest nodes to the sensed phenomenon. The former nodes perform direct routing to the cluster head in order to save their energy whereas other nodes perform multi hop routing. 


\section{Conclusions}

In this paper, we have proposed Coral-based VRR, an energy-aware ad-hoc routing protocol based on location independent identifiers. The proposed protocol takes into account nodes heterogeneity and adopts a hierarchical DHT in the VRR routing. Sensor nodes are classified according to their available energy. Coral-based VRR routing avoids energy constrained nodes and performs big logical hops among most powerful nodes.

Performance results show that Coral-based VRR outperforms VRR in terms of energy optimization, transmission delay, routing performance and network lifetime.

We believe that Coral-based VRR will be a promising routing protocol in wireless sensor networks for many applications such as objects tracking and environmental data collection because it offers both good routing performance and energy optimization.

\section{REFERENCES}

[1] Jamal N. A. and Ahmed E. K., "Routing Techniques in Wireless Sensor Networks: A Survey," IEEE Wireless Communications, vol. 11, issue 6, pp. 6-28, December 2004. http://dx.doi.org/10.1109/MWC.2004.1368893

[2] Isabel D. and Falko D., "On the lifetime of wireless sensor networks", ACM Transactions on Sensor Networks, Vol. 5, issue 1, February 2009. http://dx.doi.org/10.1145/1464420.1464425

[3] Kemal A. and Mohamed Y., "A survey on routing protocols for wireless sensor networks", Ad Hoc Network Journal, vol. 3, Issue 3, pp. 325-349, May 2005. http://dx.doi.org/10.1016/j.adhoc.2003.09.010

[4] Xuan Zhong; Khosla, R.; Khanna, G.; Bagchi, S.; Doyle, E.J.; "Data-Centric Routing in Sensor Networks: Single-hop Broadcast or Multi-hop Unicast?", IEEE 65th Vehicular Technology Conference. VTC2007-Spring. 22 - 25 April 2007, Dublin, Ireland. http://dx.doi.org/10.1109/VETECS.2007.43

[5] Wendi R. H., Anantha C., and Hari B., "Energy-efficient communication protocol for wireless sensor networks", in the Proceeding of the Hawaii International Conference System Sciences (HICSS), Maui, Hawaii, USA, 4-7 January 2000.

[6] Brad K. and H. T. Kung, "GPSR: Greedy Perimeter Stateless Routing for Wireless Networks", in the Proceedings of the 6th Annual ACM/IEEE International Conference on Mobile Computing and Networking (MobiCom '00), Boston, USA, August 2000. http://dx.doi.org/10.1145/345910.345953

[7] Matthew C., Miguel C., Edmund B. N., Greg O., and Antony R., "Virtual Ring Routing: Network Routing Inspired by DHTs", SIGCOMM06, Pisa, Italy, 2006. http://dx.doi.org/10.1145/1159913.1159954

[8] Michael J. F. and David M., "Sloppy hashing and self-organizing clusters", in second International Peer To Peer Systems Workshop (IPTPS 2003), Berkeley, CA, USA, February 2003.

[9] NS-2 Simulator, Available at: htt://www.isi.edu/nsnam/ns/

[10] Abdalkarim Awad, Lei 'Ray' Shi, Reinhard German, and Falko Dressler, "Advantages of Virtual Addressing for Efficient and Failure Tolerant Routing in Sensor Networks", Sixth International Conference on Wireless On-Demand Network Systems and Services, 2009.

[11] Sylvia R., Brad K., Scott S., Deborah E., Ramesh G., Li Y., and Fang Y., "Data-Centric Storage in Sensornets with GHT, A Geographic Hash Table", ACM/Springer Mobile 
Networks and Applications (MONET), Special Issue on Wireless Sensor Networks, vol. 8, pp. 427-442, 2003. http://dx.doi.org/10.1023/A:1024591915518

[12] Ananth Rao, Sylvia Ratnasamy, Christos Papadimitriou, Scott Shenker, Ion Stoica, "Geographic Routing without Location Information", ACM International Conference on Mobile Computing and Networking (ACMMobiCom), S. Diego, CA, 2003. http://dx.doi.org/10.1145/938985.938996

[13] Muneeb A. and Koen L., "A Case for Peer-to-Peer Network Overlays in Sensor Networks", International Workshop on Wireless Sensor Network Architecture (WWSNA), Cambridge, MA, USA, 2007.

[14] Abdalkarim Awad, Christoph Sommer, Reinhard German and Falko Dressler, "Virtual Cord Protocol (VCP): A Flexible DHT-like Routing Service for Sensor Networks", 5th IEEE Int. Conference on Mobile Ad Hoc and Sensor Systems (IEEE MASS 2008), Atlanta, Georgia, USA. Sep. 29 - Oct. 2, 2008. http://dx.doi.org/10.1109/MAHSS.2008.4660079

[15] Stephanie L. and Cauligi S. R., "PEGASIS: Power Efficient GAthering in Sensor Information Systems," in the Proceedings of the IEEE Aerospace Conference, Big Sky, Montana, March 2002. http://dx.doi.org/10.1109/AERO.2002.1035242

[16] Stephanie L. , Cauligi S. R and Krishna S., "Data Gathering in Sensor Networks using the Energy*Delay Metric", in the Proceedings of the IPDPS Workshop on Issues in Wireless Networks and Mobile Computing, San Francisco, CA, April 2001.

[17] Arati M. and Dharma P. A., "TEEN: A Protocol for Enhanced Efficiency in Wireless Sensor Networks,". 1st International Workshop on Parallel and Distributed Computing Issues in Wireless Networks and Mobile Computing, San Francisco, CA, April 2001.

[18] Manjeshwar A., Agrawal D.P, "APTEEN: A Hybrid Protocol for Efficient Routing and Comprehensive Information Retrieval in Wireless Sensor Networks," in the Proceedings of the 2nd International Workshop on Parallel and Distributed Computing Issues in Wireless Networks and Mobile computing, Ft. Lauderdale, FL, April 2002.

[19] Lakshminarayanan S. and Randy H.K., "An Architecture for Building Self Configurable Systems," in the Proceedings of IEEE/ACM Workshop on Mobile Ad Hoc Networking and Computing, Boston, MA, August 2000.

[20] Adeel A., Abid A., and Sohail J. "Energy Aware Intra Cluster Routing for Wireless Sensor Networks", international Journal of Hybrid Information Technology, Vol.3, No.1, January, 2010

\section{Copyright Disclaimer}

Copyright reserved by the author(s).

This article is an open-access article distributed under the terms and conditions of the Creative Commons Attribution license (http://creativecommons.org/licenses/by/3.0/). 